TRANSACTIONS OF THE

AMERICAN MATHEMATICAL SOCIETY

Volume 361, Number 9, September 2009, Pages 5023-5039

S 0002-9947(09)04803-X

Article electronically published on April 20, 2009

\title{
OSCILLATIONS OF A GIVEN SIZE OF SOME ARITHMETIC ERROR TERMS
}

\author{
JERZY KACZOROWSKI AND KAZIMIERZ WIERTELAK
}

\begin{abstract}
A general method of estimating the number of oscillations of a given size of arithmetic error terms is developed. Special attention is paid to the remainder terms in the prime number formula, in the Dirichlet prime number theorem for primes in arithmetic progressions and to the remainder term in the asymptotic formula for the number of square free divisors of an integer.
\end{abstract}

\section{Introduction AND STATEMENT OF RESUlTS}

Let

$$
\psi(x)=\sum_{n \leq x} \Lambda(n) \quad \text { and } \quad \pi(x)=\sum_{p \leq x} 1
$$

denote the familiar prime counting functions. A classical theorem by J. E. Littlewood [12] states that

$$
\psi(x)-x=\Omega_{ \pm}(\sqrt{x} \log \log \log x)
$$

and

$$
\pi(x)-\operatorname{li}(x)=\Omega_{ \pm}\left(\frac{\sqrt{x} \log \log \log x}{\log x}\right)
$$

as $x \rightarrow \infty$. In particular both remainders $\psi(x)-x$ and $\pi(x)-\operatorname{li}(x)$ change sign infinitely many times. Denoting by $V(T)$ the number of changes of sign of any of them in the interval $[2, T]$ we have

$$
V(T) \gg \log T
$$

(see 4]). One can speculate that the majority of changes of sign are connected with moderate oscillations, and that large oscillations such as in (1.1) and (1.2) are rather rare. The basic object of the present paper is to discuss this problem for various arithmetic error terms including $\psi(x)-x$ and $\pi(x)-\operatorname{li}(x)$. Our approach is a combination of methods from [4, [5, 8] and [1].

Received by the editors July 18, 2007 and, in revised form, January 14, 2008.

2000 Mathematics Subject Classification. Primary 11N05, 11N13, 11N25; Secondary 11M26.

Both authors were supported in part by KBN Grant N N201 148233. 
Theorem 1.1. There exist constants $H_{0}$ and $T_{0}$ such that for every function $H(T)$ defined for $T \geq T_{0}$ and satisfying $H_{0} \leq H(T) \leq \log T$ there exist points

$$
\begin{aligned}
& 2<y_{1}<y_{2}<\ldots<y_{V} \leq T, \\
& V \geq \frac{\log T}{H(T)}
\end{aligned}
$$

for which we have

$$
\left|\psi\left(y_{j}\right)-y_{j}\right| \gg \sqrt{y_{j}} \log \log H(T) \quad(j=1, \ldots, V)
$$

and

$$
\operatorname{sgn}\left(\psi\left(y_{j}\right)-y_{j}\right)=-\operatorname{sgn}\left(\psi\left(y_{j+1}\right)-y_{j+1}\right) \quad(j=1, \ldots, V-1) .
$$

To avoid repetitions let us adopt the following notation. Let $f$ and $g$ be two real-valued functions defined on an interval $I=[a, b] \subset \mathbb{R}$, and let us assume that $g$ is positive. We say that $f(x)$ has inside $I$ at least $V$ oscillations of size $g(x)$ if there exist points

$$
a \leq x_{1}<x_{2}<\ldots<x_{V} \leq b
$$

such that $\left|f\left(x_{j}\right)\right| \geq g\left(x_{j}\right)$ for $j=1, \ldots, V$ and $\operatorname{sgn} f\left(x_{j}\right)=-\operatorname{sgn} f\left(x_{j+1}\right)$ for $j=$ $1, \ldots, V-1$. In this situation we say also that $f(x)$ has at least $V-1$ changes of sign combined with oscillations of size $g(x)$.

With this notation we can restate Theorem 1 by saying that for sufficiently large $T$, the difference $\psi(x)-x$ has at least $(\log T) / H(T)$ oscillations of size $\gg \sqrt{x} \log \log H(T)$ in the interval $[2, T]$. Taking $H(T)=\exp \left((\log \log T)^{b_{0}}\right)$ with a small positive $b_{0}$ we deduce that in every interval $[2, T]$ with sufficiently large $T$, there are $\gg \log T \exp \left(-(\log \log T)^{b_{0}}\right)$ changes of sign of $\psi(x)-x$ combined with oscillations of size $\gg \sqrt{x} \log \log \log x$. We supplement in that way (1.1). In particular, surprisingly enough, we find that the number of large oscillations is not that small as one could expect. On the other extreme, taking $H(T)=\exp \exp (C)$ with arbitrarily large $C$, we deduce that $\psi(x)-x$ changes sign $\gg \log T$ times on the interval $[2, T]$ making oscillations of size $C \sqrt{x}$. This supplements (1.3).

Though (1.1), (1.2) and (1.3) are the best known results of their type, nevertheless almost surely they are not optimal. Not knowing the true behavior of them, one is left with more or less speculative conjectures, and the same applies to other remainders considered in this paper. Littlewood's theorem (1.1) is expected to be nearly the best possible. There are reasons to believe that

$$
\liminf _{x \rightarrow \infty} \frac{\psi(x)-x}{\sqrt{x}(\log \log \log x)^{2}}=-\frac{1}{2 \pi} \quad \text { and } \quad \limsup _{x \rightarrow \infty} \frac{\psi(x)-x}{\sqrt{x}(\log \log \log x)^{2}}=\frac{1}{2 \pi},
$$

as conjectured by H.L. Montgomery in [14. Concerning the number of changes of sign, numerical evidence suggests that the total number of changes of sign of $\psi(x)-x$ in the interval $[2, T]$ should be proportional to $\sqrt{T}$ if $T$ is sufficiently large; see [15]. Hence there is a large gap between (1.3) and the expected square root of $T$. This phenomenon can, at least partially, be explained by the fact that the method of counting changes of sign used in [4, 5] involves a smoothing process which probably kills the majority of changes of sign which are combined with oscillations of "small" or "moderate" size. This is in agreement with the fact that some smoothed versions of $\psi(x)-x$ oscillate in $[2, T]$ definitely less frequently than $\sqrt{T}$. For instance it was 
proved in [6] that under the Riemann Hypothesis, the Abel mean

$$
\sum_{n=2}^{\infty}(\Lambda(n)-1) e^{-n / x}
$$

has $\sim\left(\gamma_{0} / \pi\right) \log T$ changes of sign inside $[2, T]$, all combined with "large" oscillations. Unconditionally it is known that this number is $o\left(\log ^{2} T\right)$; see 7 . Finally let us remark that the above conjectures can be supported by proving corresponding results in suitable probabilistic models for primes. One has however to be aware of the fact that probabilistic models often do not accurately describe the distribution of primes (see for instance [17]), and hence we shall not discuss such types of arguments in this paper.

One has also the following version of Theorem 1.1 for primes in arithmetic progressions.

Theorem 1.2. Let $E(x)$ denote one of the error terms

$$
\psi(x, q, 1)-\frac{x}{\varphi(q)} \text { or } \psi(x, q, 1)-\psi(x, q, a),
$$

where $a \not \equiv 1(\bmod q),(a, q)=1$ and $\varphi(q)$ denotes the Euler totient function. Suppose moreover that the Dirichlet $L$-functions $L(s, \chi), \chi(\bmod q)$, do not vanish in the interval $(1 / 2,1)$. Then there exist constants $H_{0}$ and $T_{0}$ such that for every function $H(T)$ defined for $T \geq T_{0}$ and satisfying $H_{0} \leq H(T) \leq \log T, E(x)$ has in the interval $[2, T]$ at least $(\log T) / H(T)$ oscillations of size $\gg \sqrt{x} \log \log H(T)$.

Using partial summation we immediately obtain the following corollary.

Corollary 1.3. Let $E^{*}(x)$ denote one of the differences $\pi(x)-\operatorname{li}(x), \pi(x, q, 1)-$ $\frac{1}{\varphi(q)} \operatorname{li}(x)$ or $\pi(x, q, a)-\pi(x, q, 1)$ where $(a, q)=1, a \neq \equiv 1(\bmod q)$, and in the case of the second and the third difference we assume that the Dirichlet $L$-functions do not vanish in the interval $(1 / 2,1)$. Then there exist constants $H_{0}$ and $T_{0}$ such that for every function $H(T)$ defined for $T \geq T_{0}$ and satisfying $H_{0} \leq H(T) \leq \log T, E^{*}(x)$ has in the interval $[2, T]$ at least $(\log T) / H(T)$ oscillations of size

$$
\gg \frac{\sqrt{x} \log \log H(T)}{\log x} .
$$

Theorem 1.2 and Corollary 1.3 should be compared with Corollaries 1.2 and 1.3 in [11. Let us remark here that in the formulations of these corollaries in 11] the condition about non-vanishing of Dirichlet $L$-functions on $(1 / 2,1)$ was mistakenly omitted.

There are other instances where our method can successfully be applied. As in [11] and 21] let us write

$$
D_{2}(x)=\sum_{n \leq x} \theta(n),
$$

where $\theta(n)=2^{\omega(n)}$ denotes the number of square-free divisors of a positive integer $n$. The corresponding remainder term is defined as follows:

$$
E_{2}(x)=D_{2}(x)-\frac{1}{\zeta(2)} x\left(\log x+2 C-1-2 \frac{\zeta^{\prime}}{\zeta}(2)\right),
$$

where $C$ is the Euler constant. 
Upper estimates for $E_{2}(x)$ were considered by many authors (cf. [1, 2], 16, [18, [19]). The classical result is due to Mertens [13]:

$$
E_{2}(x)=O\left(x^{1 / 2} \log x\right) .
$$

It was slightly sharpened to $O\left(x^{1 / 2}\right)$ in 3 , but the exponent $1 / 2$ remains unimproved to date. However, it can be further sharpened assuming the Riemann Hypothesis. The strongest published result of this sort is

$$
E_{2}(x) \ll x^{4 / 11+\varepsilon},
$$

proved in 22, and a slight modification of the argument in 22] allows one to replace $4 / 11$ by $221 / 608$. It is expected that the right exponent is $1 / 4+\varepsilon$ for arbitrary positive $\varepsilon$. Anyway, one cannot go below $1 / 4$ as proved in [11]:

$$
E_{2}(x)=\Omega_{ \pm}\left(x^{\frac{1}{4}} \frac{(\log \log x)^{\frac{1}{2}}}{(\log \log \log x)^{\frac{3}{2}}}\right)
$$

when $x \rightarrow \infty$. We would like to supplement this result by estimating the number of oscillations of $E_{2}(x)$ of a given size. Unfortunately we are unable to achieve this unconditionally, and we have to assume the following hypothesis on the Riemann zeta function.

$(\mathrm{H})$ There exists a positive constant $B^{*}$ such that for all simple non-trivial zeros $\rho$ of the Riemann zeta function lying on the critical line $(\rho=1 / 2+i \gamma)$ we have

$$
\frac{1}{\zeta^{\prime}(\rho)} \ll|\gamma|^{B^{*}}
$$

Let us remark that hypothesis $(\mathrm{H})$ concerns exclusively simple zeros lying on the critical line. We do not exclude existence of non-trivial zeros off the critical line. Also, we do not need any extra conditions about multiple zeros, if they exist at all.

Theorem 1.4. Let us assume $(H)$. Then there exist constants $H_{0}$ and $T_{0}$ such that for every function $H(T)$ defined for $T \geq T_{0}$ and satisfying $H_{0} \leq H(T) \leq \log T$, $E_{2}(x)$ has in the interval $[2, T]$ at least $(\log T) / H(T)$ oscillations of size

$$
\gg \frac{x^{\frac{1}{4}} \log ^{\frac{1}{2}} H(T)}{(\log \log H(T))^{3 / 2}} .
$$

\section{A general theorem on functions from the Class $\mathfrak{A}$}

Following [11] let us denote by $\mathfrak{A}$ the set of all functions

$$
F(z)=\sum_{n=1}^{\infty} a_{n} e^{i w_{n} z} \quad(\Im(z)>0),
$$

where the exponents $0 \leq w_{1}<w_{2}<\ldots$ are real, and the coefficients $a_{n}, n \geq 1$, are complex, satisfying the following conditions:

(i) There exists a real number $B=B(F) \geq 0$ such that

$$
\sum_{n=2}^{\infty} \frac{\left|a_{n}\right|}{w_{n}^{B}}<\infty
$$


(ii) There exists $L_{0}=L_{0}(F) \geq 0$ such that the limit

$$
P(x)=\lim _{y \rightarrow 0^{+}} \Re F(x+i y)
$$

exists for every real $x \geq L_{0}$.

(iii) For every bounded interval $I \subset\left(L_{0}, \infty\right)$ we have

$$
\Re F(x+i y) \ll_{I} 1
$$

for $x \in I$ and $y>0$.

(iv) There exists a decreasing and continuous function $\phi:(0,1) \rightarrow \mathbb{R}, \phi(\delta) \rightarrow \infty$ as $\delta \rightarrow 0^{+}$, and two points $x_{1}, x_{2} \in \mathbb{R}$ such that

$$
\begin{aligned}
& \Re F\left(x_{1}+\delta e^{i \theta}\right) \gg \phi(\delta), \\
& \quad-\Re F\left(x_{2}+\delta e^{i \theta^{\prime}}\right) \gg \phi(\delta),
\end{aligned}
$$

as $\delta \rightarrow 0^{+}$uniformly for $\theta_{1}^{(1)}<\theta<\theta_{2}^{(1)}$ and $\theta_{1}^{(2)}<\theta^{\prime}<\theta_{2}^{(2)}$ respectively, where $0<\theta_{1}^{(j)}<\theta_{2}^{(j)}<\pi, j=1,2$, are certain fixed parameters which can depend on $F$.

(v) We have $N(T)=\#\left\{n: w_{n} \leq T\right\} \ll T \log T$ as $T \rightarrow \infty$.

Suppose that a real-valued function $f$ has in the interval $I=[a, b]$ at least $V$ oscillations of size $g(x)$. If, for certain positive $D$, the points in (1.4) are such that $x_{j+1}-x_{j} \geq D$, we say that the oscillations of $f$ are $D$-apart.

With this notation we have the following result.

Theorem 2.1. Let $F \in \mathfrak{A}$. Then there exist positive constants $H_{0}=H_{0}(F)$, $T_{0}=T_{0}(F)$ and $b_{0}=b_{0}(F)$ such that for every $H(T), T \geq T_{0}(>2)$, satisfying $H_{0} \leq H(T) \leq T$, the function $P(x)$ has in $[2, T]$ at least $T / H(T)$ oscillations of size

$$
\gg \phi\left(b_{0} \frac{(\log \log H(T))^{3}}{\log H(T)}\right)
$$

which are 1-apart.

Proof. For $j=1,2$, let

$$
\theta_{j}^{*}=\frac{\theta_{1}^{(j)}+\theta_{2}^{(j)}}{2}
$$

and

$$
z_{j}(\delta)=x_{j}+\delta e^{i \theta_{j}^{*}}
$$

where $\delta>0$. Moreover, let

$$
D\left(x_{j}, \theta_{1}^{(j)}, \theta_{2}^{(j)}\right)=\left\{z \in \mathbb{C}: z=x_{j}+r e^{i \theta}, \theta_{1}^{(j)}<\theta<\theta_{2}^{(j)}, r>0\right\} \quad(j=1,2) .
$$

There exists $b_{1}=b_{1}\left(\theta_{1}^{(1)}, \theta_{2}^{(1)}, \theta_{1}^{(2)}, \theta_{2}^{(2)}\right)>0$ such that $z_{j}(\delta)+u \in D\left(x_{j}, \theta_{1}^{(j)}, \theta_{2}^{(j)}\right)$ for $j=1,2$ and all real $u$ satisfying $|u|<b_{1} \delta$. For $N \geq 2(B+1)$ to be fixed later on, $\delta_{N}=\left(b_{1} \delta\right) / N, b_{1} \delta<1$, let us consider the following subsidiary function defined for complex $z=x+i y$ with a positive imaginary part $y$ :

$$
\begin{aligned}
F_{\delta, N}(z) & =\frac{1}{\left(2 \delta_{N}\right)^{N}} \int_{-\delta_{N}}^{\delta_{N}} \ldots \int_{-\delta_{N}}^{\delta_{N}} F\left(z+u_{1}+\ldots+u_{N}\right) d u_{1} \ldots d u_{N} \\
& =\sum_{n=1}^{\infty} a_{n} S^{N}\left(\delta_{N} w_{n}\right) e^{i w_{n} z} \quad(\Im(z)>0)
\end{aligned}
$$


where

$$
S(u)= \begin{cases}\frac{\sin u}{u} & \text { if } u \neq 0 \\ 1 & \text { otherwise }\end{cases}
$$

Let us put

$$
G=\delta_{N}^{-\frac{N}{N-B}}
$$

Then we have

$$
\begin{aligned}
\left|\sum_{w_{n}>G} a_{n} S^{N}\left(\delta_{N} w_{n}\right) e^{i w_{n} z}\right| & \leq \frac{1}{\left(\delta_{N}\right)^{N}} G^{B-N} \sum_{w_{n}>G} \frac{\left|a_{n}\right|}{w_{n}^{B}} \\
& =\sum_{w_{n}>G} \frac{\left|a_{n}\right|}{w_{n}^{B}} \ll 1 .
\end{aligned}
$$

Consequently,

$$
F_{\delta, N}(z)=\sum_{w_{n} \leq G} a_{n} S^{N}\left(\delta_{N} w_{n}\right) e^{i w_{n} z}+O(1) .
$$

Let us chose $q=\left[G^{B}\right], L_{1}=L_{0}+\max _{l=1,2}\left|x_{l}\right|+3, T>2 L_{1}\left(1+q^{N(G)}\right)$, where $N(\cdot)$ is the counting function defined in condition (v) of the definition of the class $\mathfrak{A}$, and let $\tau_{1}<\tau_{2}<\ldots<\tau_{2 V}$ be real numbers satisfying the following conditions:

$$
\left\{\begin{array}{l}
\left\|\frac{w_{n} \tau_{j}}{2 \pi}\right\| \leq \frac{1}{q} \text { for } w_{n} \leq G, j=1, \ldots, 2 V \\
\tau_{j+1}-\tau_{j} \geq L_{1} \text { for } j=1, \ldots, 2 V-1 \\
L_{1} \leq \tau_{j} \leq T-L_{0}-3 \text { for } j=1, \ldots, 2 V .
\end{array}\right.
$$

Then for $z=x+i y, y>0$, we have

$$
\begin{aligned}
\left|F_{\delta, N}(z)-F_{\delta, N}\left(z+\tau_{j}\right)\right| & \ll \sum_{w_{n} \leq G}\left|a_{n}\right|\left|e^{i w_{n} \tau_{j}}-1\right|+1 \\
& \ll \frac{1}{q} \sum_{w_{n} \leq G}\left|a_{n}\right|+1 \ll \frac{1}{q} G^{B}+1 \ll 1 .
\end{aligned}
$$

Let $x_{0}=L_{0}+2$. Using (2.3) and condition (iii) from the definition of the class $\mathfrak{A}$ we see that for $y>0$,

$$
\Re F_{\delta, N}\left(x_{0}+\tau_{j}+i y\right) \ll 1 \quad(j=1, \ldots, 2 V),
$$

where the implied constant depends only on $L_{0}$. On the other hand, using (2.3) and condition (iv) from the definition of the class $\mathfrak{A}$ we see that

$$
\Re F_{\delta, N}\left(z_{1}(\delta)+\tau_{j}\right) \geq \Re F_{\delta, N}\left(z_{1}(\delta)\right)+O(1) \gg \phi(\delta)
$$

and

$$
-\Re F_{\delta, N}\left(z_{2}(\delta)+\tau_{j}\right) \gg \phi(\delta)
$$

for $j=1, \ldots, 2 V$ and sufficiently small $\delta>0$.

Let us consider the rectangles with vertices

$$
\begin{gathered}
x_{0}+\tau_{2 j-1}+i y_{0}, \quad x_{0}+\tau_{2 j-1}, \quad x_{0}+\tau_{2 j}, \quad x_{0}+\tau_{2 j}+i y_{0} \\
(j=1, \ldots, V)
\end{gathered}
$$


where $y_{0}>0$ is chosen so large that $\left|\Re F_{\delta, N}\left(x+i y_{0}\right)\right| \leq 1$ for all real $x$. This is possible since $F_{\delta, N}(x+i y)$ tends to zero as $y \rightarrow \infty$ uniformly in $x \in \mathbb{R}$. From our choice of $x_{0}$ and $L_{1}$ we have for $l=1,2$ and $j=1, \ldots, V$,

$$
\begin{aligned}
\Re z_{l}(\delta)+\tau_{2 j} & \geq \Re z_{l}(\delta)+\tau_{2 j-1}+L_{1} \\
& >\left(\min _{l=1,2} x_{l}-1\right)+\tau_{2 j-1}+\left(L_{0}+\max _{l=1,2}\left|x_{l}\right|+3\right) \\
& \geq x_{0}+\tau_{2 j-1}
\end{aligned}
$$

and similarly

$$
\Re z_{l}(\delta)+\tau_{2 j}<x_{0}+\tau_{2 j} .
$$

Applying the maximum principle for the harmonic function $\Re F_{\delta, N}(z)$ inside the above defined rectangles and using estimates (2.4) - (2.6) we obtain for $j=1, \ldots, V$,

$$
\max _{x_{0}+\tau_{2 j-1} \leq x \leq x_{0}+\tau_{2 j}} \Re F_{\delta, N}(x) \geq \Re F_{\delta, N}\left(z_{1}(\delta)+\tau_{2 j}\right) \gg \phi(\delta)
$$

and

$$
-\min _{x_{0}+\tau_{2 j-1} \leq x \leq x_{0}+\tau_{2 j}} \Re F_{\delta, N}(x) \gg \phi(\delta) .
$$

On the other hand, using the Lebesgue Bounded Convergence Theorem and condition (iii) from the definition of the class $\mathfrak{A}$, after taking real parts in (2.1), we can pass to the limit as $y \rightarrow 0^{+}$. Therefore for real $x \geq L_{0}+1$ we have

$$
\Re F_{\delta, N}(x)=\frac{1}{\left(2 \delta_{N}\right)^{N}} \int_{-\delta_{N}}^{\delta_{N}} \ldots \int_{-\delta_{N}}^{\delta_{N}} P\left(x+u_{1}+\ldots+u_{N}\right) d u_{1} \ldots d u_{N} .
$$

Consequently

$$
\phi(\delta) \ll \max _{x_{0}+\tau_{2 j-1} \leq x \leq x_{0}+\tau_{2 j}} \Re F_{\delta, N}(x) \leq \max _{x_{0}+\tau_{2 j-1}-1 \leq x \leq x_{0}+\tau_{2 j}+1} P(x),
$$

and similarly

$$
\phi(\delta) \ll-\min _{x_{0}+\tau_{2 j-1}-1 \leq x \leq x_{0}+\tau_{2 j}+1} P(x) .
$$

Picking up points $y_{j} \in\left(x_{0}+\tau_{2 j-1}-1, x_{0}+\tau_{2 j}+1\right), j=1, \ldots, V$, for which $P\left(y_{j}\right)$ are large in absolute value but the signs sgn $P\left(y_{j}\right)$ alternate, we conclude that $P(x)$ has in the interval $[2, T]$ at least $V$ oscillations of size $\gg \phi(\delta)$ which are $L_{1} \geq 1$ apart. Hence in order to conclude the proof it suffices to show that there are sufficiently many solutions of (2.2) and to choose $\delta$ and $N$ properly.

Let us put

$$
N=\left[\log \frac{1}{b_{1} \delta}\right] .
$$

Then for sufficiently small $\delta>0$ we have $N \geq 2(B+1)$, as required. Moreover, by the classical Dirichlet theorem on Diophantine approximations in the version given at the end of Section 8.2 in [20, we see that there exist $\tau_{1}, \ldots, \tau_{2 V}$ with

$$
V=\left[\frac{T-L_{0}-3}{2 L_{1} q^{N(G)}}\right]>\frac{T}{4 L_{1} q^{N(G)}}
$$

satisfying (2.2). By condition (v) from the definition of the class $\mathfrak{A}$ we have $N(G) \leq$ $c_{1} G \log G$ for a certain constant $c_{1}$ depending on $F$. Hence we have

$$
q^{N(G)} \leq \exp \left(c_{1} B G \log ^{2} G\right) \leq \exp \left(c_{1} c_{2} \frac{\log ^{3} \frac{1}{b_{1} \delta}}{b_{1} \delta}\right)
$$


where $c_{2}$ is a constant depending only on $B$. Consequently, choosing

$$
\delta=\frac{2 c_{1} c_{2}}{b_{1}} \frac{(\log \log H(T))^{3}}{\log H(T)},
$$

we obtain for sufficiently large $H(T)$,

$$
V>\frac{T}{4 L_{1}(H(T))^{1 / 2}}>\frac{T}{H(T)}
$$

and the result follows.

\section{Proof of Theorem 1}

We shall need the following lemma.

Lemma 3.1. Let $f(x)$ be real for $x>0$ and suppose that the integral

$$
F(s)=\int_{0}^{\infty} f(x) x^{-s-1} d x
$$

converges absolutely for $\sigma>\sigma_{0}$ and has meromorphic continuation to a half-plane $\sigma>\theta$ for certain $\theta<\sigma_{0}$. Suppose that $F(s)$ is not holomorphic for $\sigma>\theta$ but is holomorphic on the segment $\left(\theta, \sigma_{0}\right]$ of the real axis. Then for sufficiently large $T$, $f(x)$ has in the interval $(0, T]$ at least $\gg \log T$ oscillations of size $x^{\theta}$.

Proof. This is a special case of Theorem 1 in 8 .

Let us apply this result to

$$
f(x)= \begin{cases}0 & \text { if } 0<x<1 \\ \psi(x)-x & \text { if } x \geq 1\end{cases}
$$

In that case

$$
F(s)=-\frac{1}{s} \frac{\zeta^{\prime}}{\zeta}(s)-\frac{1}{s-1}
$$

and hence we can take $\sigma_{0}=1$. If the Riemann Hypothesis is not true, the assumptions of Lemma 3.1 are satisfied with a certain $\theta>1 / 2$. Consequently for sufficiently large $T, \psi(x)-x$ has in the interval $[2, T]$ at least $\gg \log T$ oscillations of size $x^{\theta}$. Since the number of such oscillations in $[2, \log \log T]$ is $O(\log \log T)$, we see that in the interval $[\log \log T, T]$ there are at least

$$
\gg \log T+O(\log \log T) \gg \log T \gg \frac{\log T}{H(T)}
$$

oscillations of size

$$
x^{\theta} \geq \sqrt{x}(\log \log T)^{\theta-\frac{1}{2}} \geq \sqrt{x} \log \log H(T),
$$

and Theorem 1.1 follows in that case.

If the Riemann Hypothesis is true, we consider the following function defined for complex $z$ with positive imaginary parts:

$$
F(z)=\sum_{\gamma>0} \frac{1}{\rho} e^{i \gamma z},
$$

where the summation is taken over all non-trivial zeros $\rho=(1 / 2)+i \gamma$ of the Riemann zeta function with $\gamma>0$. From [9] (see also [10]) we have that $F \in \mathfrak{A}$. In fact, conditions (i) $-(\mathrm{v})$ are satisfied with $B=1+\varepsilon$ for every $\varepsilon>0, L_{0}=1$, 
$x_{1}=x_{2}=0, \phi(\delta)=\log (1 / \delta), 0<\theta_{1}^{(1)}<\theta_{2}^{(1)}<(\pi / 2)$ and $(\pi / 2)<\theta_{1}^{(2)}<\theta_{2}^{(2)}<\pi$. Moreover, in that case we have for $x>0$,

$$
P(x)=-\frac{1}{2}\left(\psi_{0}\left(e^{x}\right)-e^{x}\right) e^{-\frac{x}{2}}-\frac{1}{4} e^{-\frac{x}{2}} \log \left(1-e^{-2 x}\right)+B_{0} e^{-\frac{x}{2}},
$$

where

$$
\psi_{0}(x)=\frac{1}{2}(\psi(x-0)+\psi(x+0))
$$

and $B_{0}$ is a constant. Applying Theorem 2.1 to $F(z)$ and changing variables we immediately obtain the assertion of Theorem 1.1

\section{Proof of Theorem 2}

One proceeds similarly as in the proof of Theorem 1.1 replacing the Riemann zeta function by Dirichlet $L$-functions with characters $\chi(\bmod q)$. In the case when the Generalized Riemann Hypothesis is not true we apply Lemma 3.1 to $f(x)=E(x)$ $(x \geq 1), f(x)=0(0<x<1)$, and otherwise we apply Theorem 2.1 to the functions $(\Im(z)>0)$

$$
F_{1}(z)=\frac{e^{-z / 2}}{\varphi(q)} \sum_{\chi(\bmod q)} K(z, \chi)
$$

or

$$
F_{2}(z)=\frac{e^{-z / 2}}{\varphi(q)} \sum_{\chi(\bmod q)}(\overline{\chi(a)}-1) K(z, \chi)
$$

where $K(z, \chi)$ are $K$-functions studied in [9] (compare also [1]).

\section{Proof of Theorem 3}

Let us assume first that the Riemann Hypothesis is not true. In that case we apply Lemma 3.1 to the function $f(x)$ defined for positive real $x$ as follows:

$$
f(x)= \begin{cases}E_{2}(x) & \text { if } x \geq 1 \\ 0 & \text { if } 0<x<1\end{cases}
$$

Due to (1.5), the corresponding Mellin transform defined by (3.1) converges absolutely for $\sigma>1 / 2$. Easy computations show that for such $\sigma$ one has

$$
F(s)=\frac{1}{s} \frac{\zeta^{2}(s)}{\zeta(2 s)}-\frac{1}{\zeta(2)} \frac{1}{(s-1)^{2}}-\frac{2 C-1-2\left(\zeta^{\prime} / \zeta\right)(2)}{\zeta(2)} \frac{1}{(s-1)} .
$$

Hence we can apply Lemma 3.1 with $\sigma_{0}=1 / 2$ and suitable $\theta \in(1 / 4,1 / 2)$. In that way we find that for sufficiently large $T, f(x)$ has in $[2, T]$ at least $\gg \log T$ oscillations of size $x^{\theta}$, and Theorem 1.4 follows by the analogous arguments as in the first part of the proof of Theorem 1.1. The case when the Riemann Hypothesis is true is more involved and shall be considered in the two subsequent subsections.

5.1. The case of multiple zeros under the Riemann Hypothesis. We shall need the following lemmas.

Lemma 5.1. Under the Riemann Hypothesis we have

$$
\zeta(s) \ll|t|^{\max \left(0, \frac{1}{2}-\sigma\right)+\varepsilon} \quad(|t| \geq 1) .
$$


Proof. This follows from the fact that the Riemann Hypothesis implies the Lindelöf conjecture and from the well-known convexity bound; compare [20, Chapter XIII.

Lemma 5.2. Under the Riemann Hypothesis there exist positive constants $t_{0}$ and A such that for

$$
\frac{1}{4}<\sigma \leq \frac{1}{4}+\frac{1}{2 \log \log |t|}
$$

and $|t| \geq t_{0}$ we have

$$
\frac{\zeta^{2}(s)}{\zeta(2 s)} \ll|t| \exp \left(A \frac{\log |t|}{\log \log |t|} \log \frac{1}{\left(\sigma-\frac{1}{4}\right) \log \log |t|}\right) .
$$

Proof. By Lemma 5.1 we have

$$
\zeta^{2}(s) \ll|t|^{(1 / 2)+\varepsilon} \ll|t|
$$

for $\sigma \geq 1 / 4$ and sufficiently large $|t|$. Moreover, from Theorem 14.14(B) in [20] we have

$$
\frac{1}{|\zeta(s)|} \leq \exp \left(A \frac{\log |t|}{\log \log |t|} \log \frac{2}{\left(\sigma-\frac{1}{2}\right) \log \log |t|}\right)
$$

for sufficiently large $|t|$ and

$$
\frac{1}{2}<\sigma \leq \frac{1}{2}+\frac{1}{\log \log |t|} .
$$

Changing variables in (5.3) and combining it with (5.2) we obtain the assertion.

Lemma 5.3. Under the Riemann Hypothesis every interval $[T, T+1], T>0$, contains a value of $t$ such that

$$
\frac{\zeta^{2}(s)}{\zeta(2 s)} \ll t
$$

for $1 / 8 \leq \sigma \leq 1$.

Proof. Every interval $[T, T+1], T>0$, contains a value of $t$ such that

$$
\frac{1}{\zeta(2 s)} \ll t^{\varepsilon}
$$

for $(1 / 8) \leq \sigma \leq 1$. For $\sigma \geq 1 / 4$ this easily follows from Theorem 14.16 in [20] and for the remaining $\sigma$ by the functional equation of the Riemann zeta function. Combining this estimate with (5.1) we easily obtain the assertion.

In order to study oscillations of $E_{2}(x)$ we shall apply a modification of the method introduced in 4 and then developed in 8 . Let $\mathcal{X}$ denote the linear space of realvalued functions $f(x)$ defined for positive $x$ which are Lebesgue locally integrable and such that

$$
\int_{0}^{1}|f(t)||\log t|^{N} \frac{d t}{t}<\infty
$$

for every integer $N \geq 1$. Moreover, let $\delta_{1}: \mathcal{X} \rightarrow \mathcal{X}$ be defined as follows:

$$
\delta_{1}(f)(x)=\int_{0}^{x} \frac{f(t)}{t} d t \quad(x>0, f \in \mathcal{X})
$$

and for positive integers $k$, let $\delta_{k}$ denote the $k$-fold iteration of $\delta_{1}$ :

$$
\delta_{k}=\delta_{1} \circ \ldots \circ \delta_{1} \quad(k \text { times }) .
$$


The basis of our analysis is the following elementary lemma.

Lemma 5.4. Let $f \in \mathcal{X}$ and let $I=[a, b] \subset[2, \infty)$. Suppose that $\delta_{1}(f)$ has in $I$ at least $V$ oscillations of size $\beta x^{\theta}(\log x)^{\lambda}$, where $\beta, \theta$ and $\lambda$ are positive constants. Then $f$ has in $I$ at least $V-1$ oscillations of size $\theta \beta x^{\theta} \log ^{\lambda} x$.

Proof. Let $a \leq x_{1}<x_{2}<\ldots<x_{V} \leq b$ be such that $\left|\delta_{1}(f)\left(x_{j}\right)\right| \geq \beta x_{j}^{\theta}\left(\log x_{j}\right)^{\lambda}$ for $j=1, \ldots, V$ and $\operatorname{sgn} \delta_{1}(f)\left(x_{j}\right)=-\operatorname{sgn} \delta_{1}(f)\left(x_{j+1}\right)$ for $j=1, \ldots, V-1$. Let $y_{j} \in\left(x_{j}, x_{j+1}\right)(j=1, \ldots, V-1)$ be such that $\delta_{1}(f)\left(y_{j}\right)=0$. We have

$$
\delta_{1}(f)\left(x_{j+1}\right)=\int_{y_{j}}^{x_{j+1}} \frac{f(t)}{t} d t
$$

Consequently, if $\operatorname{sgn}\left(\delta_{1}(f)\left(x_{j+1}\right)\right) f(t)<\theta \beta t^{\theta}(\log t)^{\lambda}$ for $t \in\left(y_{j}, x_{j+1}\right)$, then

$$
\left|\delta_{1}(f)\left(x_{j+1}\right)\right|<\beta x_{j+1}^{\theta}\left(\log x_{j+1}\right)^{\lambda}
$$

a contradiction. Hence there exist points $\xi_{j} \in\left(y_{j}, x_{j+1}\right), j=1, \ldots, V-1$, such that $\left|f\left(\xi_{j}\right)\right| \geq \theta \beta \xi_{j}^{\theta}\left(\log \xi_{j}\right)^{\lambda}$ and $\operatorname{sgn} f\left(\xi_{j}\right)=\operatorname{sgn} \delta_{1}(f)\left(x_{j+1}\right)$ for $j=1, \ldots, V-1$, and the lemma follows.

Lemma 5.5. Suppose that the Riemann Hypothesis is true and that the Riemann zeta function has at least one multiple non-trivial zero. Then for every real $\alpha$ and $\lambda$ satisfying $0<\alpha, \lambda<1$ and for all $T \geq T_{0}(\alpha, \lambda)$, the remainder $E_{2}(x)$ has in the interval $\left[T^{\alpha}, T\right]$ at least $\gg \log T$ oscillations of size $x^{1 / 4} \log ^{\lambda} x$, where the implied constant depends only on $\alpha$.

Proof. It is easy to see that $E_{2} \in \mathcal{X}$ and for integers $n \geq 2$ we have

$$
\delta_{n-1}\left(E_{2}\right)(x)=\frac{1}{2 \pi i} \int_{3 / 4-i \infty}^{3 / 4+i \infty} \frac{\zeta^{2}(s)}{\zeta(2 s)} \frac{x^{s}}{s^{n}} d s
$$

Let $\rho_{1}=(1 / 2)+i \gamma_{1}$ denote the "lowest" multiple zero of the Riemann zeta function. Numerical computations show that $\gamma_{1}$ should be very large and we shall use this later on without further comments. In fact for our purposes an easy bound such as $\gamma_{1}>100$ (compare [20]) is completely sufficient.

We fix a positive number

$$
G>\max \left(t_{0}, \exp \exp \left(\frac{6 A \log \left|\rho_{1}\right|}{1-\lambda}\right)\right)
$$

where $t_{0}$ and $A$ have the same meaning as in Lemma 5.2 , in such a way that

$$
\left|\frac{\zeta^{2}(s)}{\zeta(2 s)}\right| \ll G
$$

for $s=\sigma+i G, 1 / 8 \leq \sigma \leq 1$. This is possible according to Lemma 5.3 . 
For $x \geq \log G$ we replace the line of integration in (5.4) by the curve $\mathcal{C}$ consisting of the following parts:

$$
\begin{array}{llrl}
\mathcal{C}_{1}: & s=\frac{1}{4}+\frac{1}{2 \log \log |t|}+i t, & -\infty<t \leq-e^{x}, \\
\mathcal{C}_{2}: & s=\frac{1}{4}+\frac{1}{2 \log x}+i t, & -e^{x} \leq t \leq-G, \\
\mathcal{C}_{3}: & s=\sigma-i G, & \frac{1}{4}+\frac{1}{2 \log x} \geq \sigma \geq \frac{1}{8}, \\
\mathcal{C}_{4}: & s=\frac{1}{8}+i t, & -G \leq t \leq G, \\
\mathcal{C}_{5}: & s=\sigma+i G, & \frac{1}{8} \leq \sigma \leq \frac{1}{4}+\frac{1}{2 \log x} \\
\mathcal{C}_{6}: & s=\frac{1}{4}+\frac{1}{2 \log x}+i t, & G \leq t \leq e^{x}, \\
\mathcal{C}_{7}: & s=\frac{1}{4}+\frac{1}{2 \log \log |t|}+i t, & e^{x} \leq t<\infty .
\end{array}
$$

We have

$$
\begin{aligned}
\delta_{n-1}\left(E_{2}\right)(x) & =\sum_{|\gamma|<2 G} \operatorname{Res}_{s=\rho / 2}\left(\frac{\zeta^{2}(s)}{\zeta(2 s)} \frac{x^{s}}{s^{n}}\right)+\frac{1}{2 \pi i} \int_{\mathcal{C}} \frac{\zeta^{2}(s)}{\zeta(2 s)} \frac{x^{s}}{s^{n}} d s \\
& =\mathcal{R}(x, n)+\mathcal{J}(x, n),
\end{aligned}
$$

say. We split the last integral according to $\mathcal{C}=\mathcal{C}_{1} \cup \ldots \cup \mathcal{C}_{7}$ and write

$$
\mathcal{J}(x, n)=\sum_{j=1}^{7} \mathcal{J}_{j},
$$

say. Using Lemma 5.2 we have

$$
\mathcal{J}_{1}, \mathcal{J}_{7} \ll x^{\frac{1}{4}} \int_{e^{x}}^{\infty} \frac{d t}{t^{n-2}} \ll x^{1 / 4} e^{-x} \ll 1
$$

if $n \geq 4$. Similarly,

$$
\mathcal{J}_{2}, \mathcal{J}_{6} \ll x^{1 / 4} \int_{G}^{e^{x}} \exp \left(A \frac{\log t}{\log \log t} \log \log x-(n-1) \log t\right) d t \ll x^{1 / 4}
$$

if

$$
n \geq 3+A \frac{\log \log x}{\log \log G}
$$

Using (5.6) we obtain

$$
\mathcal{J}_{3}, \mathcal{J}_{5} \ll x^{1 / 4}
$$

Finally we also have

$$
\mathcal{J}_{4} \ll x^{1 / 8} 8^{n} \leq x^{1 / 8} e^{3 n} \leq x^{1 / 4}
$$

if $n \leq(1 / 24) \log x$. Consequently we have

$$
\delta_{n-1}\left(E_{2}\right)(x)=\mathcal{R}(x, n)+O\left(x^{1 / 4}\right)
$$

if

$$
4+A \frac{\log \log x}{\log \log G} \leq n \leq \frac{1}{24} \log x .
$$


For simplicity we write $\rho=2 \omega_{\rho}$. With this notation we have

$$
\operatorname{Res}_{s=\rho / 2}\left(\frac{\zeta^{2}(s)}{\zeta(2 s)} \frac{x^{s}}{s^{n}}\right)=\frac{x^{\omega_{\rho}}}{\omega_{\rho}^{n}} P_{\omega_{\rho}}(\log x),
$$

where $P_{\omega_{\rho}}$ is a polynomial with complex coefficients of degree $\operatorname{deg} P_{\omega_{\rho}}=\operatorname{ord} \rho-1$. Writing

$$
P_{\omega_{\rho}}(X)=\sum_{l=0}^{\operatorname{ord} \rho-1} A_{\text {ord } \rho-1-l} X^{l}
$$

we have

$$
A_{l}=A_{l}(\rho, n) \ll_{\rho} n^{l}
$$

and

$$
A_{0}=A_{0}\left(\omega_{\rho}\right)=\frac{1}{(\operatorname{ord} \rho-1) !} \lim _{s \rightarrow \omega_{\rho}}\left\{\left(s-\omega_{\rho}\right)^{\operatorname{ord} \rho} \frac{\zeta^{2}(s)}{\zeta(2 s)}\right\} \neq 0 .
$$

Consequently

$$
P_{\omega_{\rho}}(\log x)=A_{0}\left(\omega_{\rho}\right)(\log x)^{\operatorname{ord} \rho-1}\left(1+O\left(\frac{n}{\log x}\right)\right),
$$

where the implied constant may depend on $\rho$.

In order to analyze finer structure of $\mathcal{R}(x, n)$ we define inductively the following sequence of non-trivial zeros of the Riemann zeta function. We put $\rho_{0}=1 / 2+$ $i 14.13 \ldots$, the "lowest" zeta zero. If $\rho_{0}, \ldots, \rho_{m}$ are already defined, we define $\rho_{m+1}$ as the zero $\rho^{\prime}=1 / 2+i \gamma^{\prime}$ with the least $\gamma^{\prime}>\gamma_{m}$ such that

$$
\text { ord } \rho^{\prime}>\operatorname{ord} \rho
$$

for all zeros $\rho=1 / 2+i \gamma$ with $|\gamma|<\gamma^{\prime}$. If there are no zeros $\rho^{\prime}$ satisfying (5.10), $\rho_{m+1}$ is undefined and the sequence $\left(\rho_{m}\right)_{m=0}^{\mathcal{M}}$ is finite; otherwise we write $\mathcal{M}=\infty$. Since we assume that the Riemann zeta function has at least one multiple zero, we have $\mathcal{M} \geq 1$, and in particular $\rho_{1}=1 / 2+i \gamma_{1}$ is defined and the notation is consistent with that introduced at the beginning of the proof. We put $\mu_{l}=\operatorname{ord} \rho_{l}$ for every finite $0 \leq l \leq \mathcal{M}$. Obviously $\mu_{0}=1$ and $\mu_{l} \geq l+1$ for $l \geq 1$.

Let $\omega_{0}, \ldots, \omega_{M}$ be the initial terms of the sequence $\left(\omega_{m}\right)_{m=0}^{\mathcal{M}}$ satisfying

$$
\omega_{j}=\omega_{\rho_{j}}, \quad 0<\Im \omega_{j}<G .
$$

For every $\omega_{\rho}, 0<\Im \omega_{\rho}<G$, there exists exactly one $j \in\{0, \ldots, M\}$ such that $\operatorname{ord} \rho \leq \mu_{j}$ and $\mu_{j}$ is as small as possible. In such a case we write $\omega_{\rho} \in \Gamma_{j}$. With this notation the sum of the residues in (5.7) equals

$$
\begin{aligned}
& 2 \Re \sum_{j=0}^{M} \sum_{\omega_{\rho} \in \Gamma_{j}} \frac{x^{\omega_{\rho}}}{\omega_{\rho}^{n}} P_{\omega_{\rho}}(\log x) \\
& =2 \Re \sum_{j=0}^{M}\left\{\frac{x^{\omega_{j}}}{\omega_{j}^{n}} P_{\omega_{j}}(\log x)+\sum_{\substack{\omega_{\rho} \in \Gamma_{j} \\
\omega_{\rho} \neq \omega_{j}}} \frac{x^{\omega_{\rho}}}{\omega_{\rho}^{n}} P_{\omega_{\rho}}(\log x)\right\} \\
& =2 \Re \sum_{j=0}^{M} \frac{x^{\omega_{j}}}{\omega_{j}^{n}} P_{\omega_{j}}(\log x)\left\{1+O\left(\sum_{\substack{\omega_{\rho} \in \Gamma_{j} \\
\omega_{\rho} \neq \omega_{j}}}\left|\frac{\omega_{j}}{\omega_{\rho}}\right|^{n}\left|\frac{P_{\omega_{\rho}}(\log x)}{P_{\omega_{j}}(\log x)}\right|\right) .\right.
\end{aligned}
$$


For sufficiently large $x$ and

$$
n \leq b_{0} \log x
$$

with a sufficiently small positive $b_{0}=b_{0}(G)$ we have, using (5.9),

$$
\left|P_{\omega_{j}}(\log x)\right| \gg(\log x)^{\mu_{j}-1}
$$

and

$$
P_{\omega_{\rho}}(\log x) \ll(\log x)^{\mu_{j}-1}
$$

if $\omega_{\rho} \in \Gamma_{j}$, where the implied constants may depend on $G$. Moreover, let $0<\Delta<$ $1 / 2$ be such that

$$
\left|\frac{\omega_{j}}{\omega_{\rho}}\right| \leq e^{-\Delta}
$$

for all $\omega_{\rho} \in \Gamma_{j}, \omega_{\rho} \neq \omega_{j},|\gamma|<2 G, j=1, \ldots, M$. Of course $\Delta=\Delta(G)$. With this notation the remainder term in (5.11) is

$$
\ll \sum_{\substack{\omega_{\rho} \in \Gamma_{j} \\ \omega_{\rho} \neq \omega_{j}}}\left|\frac{\omega_{j}}{\omega_{\rho}}\right|^{n} \ll e^{-\Delta n} .
$$

Let us write for simplicity $c_{j}=A_{0}\left(\omega_{j}\right)$ and assume that $n$ satisfies the following condition:

$$
n e^{\Delta n} \leq \log x .
$$

Then using (5.9) and (5.13) we obtain

$$
\begin{aligned}
\mathcal{R}(x, n) & =2 \Re \sum_{j=1}^{M} c_{j} \frac{x^{\omega_{j}}}{\omega_{j}^{n}} \log ^{\mu_{j}-1} x\left(1+O\left(e^{-\Delta n}\right)\right) \\
& =2 x^{1 / 4} \sum_{j=1}^{M} \frac{\left|c_{j}\right|}{\left|\omega_{j}\right|^{n}} \log ^{\mu_{j}-1} x\left(\cos \left(\Im\left(\omega_{j}\right) \log x+\phi_{j}(n)\right)+O\left(e^{-\Delta n}\right)\right),
\end{aligned}
$$

where $\phi_{j}(n)=-n \arg \omega_{j}+\arg c_{j}$.

Let $T^{\alpha} \leq x \leq T$ and let

$$
\vartheta_{0}=\frac{2 A \log \left|\rho_{1}\right|}{\log \log G}
$$

We fix $n$ by putting

$$
n=\left[\frac{\vartheta}{\log \left|\rho_{1}\right|} \log \log T\right],
$$

where $\vartheta \in\left(\vartheta_{0}, 2 \vartheta_{0}\right)$ will be specified later on. Let us observe that for such an $n$ we have

$$
n \geq \frac{2 A}{\log \log G} \log \log T-1 \geq A \frac{\log \log x}{\log \log G}+4
$$

for $T \geq T_{0}(A, G)$, and hence the lower estimate in (5.8) is satisfied. It is easy to see that the upper estimate in (5.8) as well as (5.12) is also satisfied for $T \geq T_{0}(\alpha, A, G)$. Finally,

$$
n e^{\Delta n} \leq \frac{2 \vartheta_{0}}{\log \left|\rho_{1}\right|}(\log \log T)(\log T)^{\frac{2 \Delta \vartheta_{0}}{\log \left|\rho_{1}\right|}} \leq \log x
$$


for sufficiently large $T$ because

$$
\frac{2 \Delta \vartheta_{0}}{\log \left|\rho_{1}\right|}=\frac{4 \Delta A}{\log \log G}<\frac{2 \Delta(1-\lambda)}{3 \log \left|\rho_{1}\right|}<1
$$

according to (5.5) and the fact that $\left|\rho_{1}\right|>100$. Consequently (5.14) is satisfied as well. Hence for $T, x$ and $n$ as above we have

$$
\mathcal{R}(x, n)=2 x^{1 / 4} \sum_{j=1}^{M} \frac{\left|c_{j}\right|}{\left|\omega_{j}\right|^{n}} \log ^{\mu_{j}-1} x\left(\cos \left(\Im\left(\omega_{j}\right) \log x+\phi_{j}(n)\right)+o(1)\right) .
$$

We chose the dominating term in the last sum. Let us write

$$
\Lambda_{j}(\vartheta)=\mu_{j}-1-\vartheta \frac{\log \left|\omega_{j}\right|}{\log \left|\rho_{1}\right|} .
$$

Then

$$
\frac{\log ^{\mu_{j}-1} x}{\left|\omega_{j}\right|^{n}}=\exp \left(\Lambda_{j}(\vartheta) \log \log x+O_{\alpha, G}(1)\right) .
$$

Since for $j \neq j^{\prime}$ we have $\log \left|\omega_{j}\right| \neq \log \left|\omega_{j^{\prime}}\right|$, there exists $\vartheta^{*} \in\left(\vartheta_{0}, 2 \vartheta_{0}\right)$ such that $\Lambda_{j}\left(\vartheta^{*}\right) \neq \Lambda_{j^{\prime}}\left(\vartheta^{*}\right)$ for all $j \neq j^{\prime}, 1 \leq j, j^{\prime} \leq M$. We put $\vartheta=\vartheta^{*}$ in (5.16), and define $D_{0}$ and $D_{1}$ as follows:

$$
\begin{gathered}
D_{0}=\min _{j \neq j^{\prime}}\left|\Lambda_{j}\left(\vartheta^{*}\right)-\Lambda_{j^{\prime}}\left(\vartheta^{*}\right)\right|, \\
D_{1}=\max _{1 \leq j \leq M} \Lambda_{j}\left(\vartheta^{*}\right)=\Lambda_{j_{1}}\left(\vartheta^{*}\right) .
\end{gathered}
$$

Then we have

$$
D_{1} \geq \Lambda_{1}\left(\vartheta^{*}\right)=\mu_{1}-1-\vartheta^{*} \frac{\log \left|\omega_{1}\right|}{\log \left|\rho_{1}\right|} \geq 1-2 \vartheta_{0} .
$$

Consequently

$$
\frac{\log ^{\mu_{j_{1}}-1} x}{\left|\omega_{j_{1}}\right|^{n}} \gg \log ^{D_{1}} x \gg \log ^{1-2 \vartheta_{0}} x .
$$

Gathering the above estimates we obtain

$$
\begin{aligned}
\delta_{n-1}\left(E_{2}\right)(x)= & 2 x^{1 / 4}\left|c_{j_{1}}\right| \frac{\log ^{\mu_{j_{1}}-1} x}{\left|\omega_{j_{1}}\right|^{n}}\left\{\cos \left(\Im\left(\omega_{j_{1}}\right) \log x+\phi_{j_{1}}(n)\right)+o(1)\right. \\
& \left.+\sum_{\substack{1 \leq j \leq M \\
j \neq j_{1}}} \frac{\left|c_{j}\right|}{\left|c_{j_{1}}\right|} \exp \left(\left(\Lambda_{j}\left(\vartheta^{*}\right)-\Lambda_{j_{1}}\left(\vartheta^{*}\right)\right) \log \log x+O_{\alpha, G}(1)\right)\right\} \\
& +O\left(x^{\frac{1}{4}}\right) \\
= & 2 x^{1 / 4}\left|c_{j_{1}}\right| \frac{\log ^{\mu_{j_{1}}-1} x}{\left|\omega_{j_{1}}\right|^{n}}\left\{\cos \left(\Im\left(\omega_{j_{1}}\right) \log x+\phi_{j_{1}}(n)\right)+o(1)\right. \\
& +O\left(M \exp \left(-D_{0} \log \log x+O_{\alpha, G}(1)\right)\right)+O\left(x^{\frac{1}{4}}\right) \\
= & 2 x^{1 / 4}\left|c_{j_{1}}\right| \frac{\log ^{\mu_{j_{1}}-1} x}{\left|\omega_{j_{1}}\right|^{n}}\left\{\cos \left(\Im\left(\omega_{j_{1}}\right) \log x+\phi_{j_{1}}(n)\right)+o(1)\right\} .
\end{aligned}
$$

Hence for sufficiently large $T$, the function $\delta_{n-1}\left(E_{2}\right)(x)$ has in the interval $\left[T^{\alpha}, T\right]$ at least $\gg \log T$ oscillations of size $\gg x^{1 / 4}(\log x)^{1-2 \vartheta_{0}}$, and the implied constant in the first estimate depends only on $\alpha$. Applying Lemma 5 we see that $E_{2}(x)$ has in 
$\left[T^{\alpha}, T\right]$ at least $\gg \log T+O(n) \gg \log T$ oscillations of size $\gg 4^{-n} x^{1 / 4}(\log x)^{1-2 \vartheta_{0}} \gg$ $x^{1 / 4}(\log x)^{1-3 \vartheta_{0}}$, and the lemma follows since according to (5.5) and (5.15) we have $1-3 \vartheta_{0}>\lambda$.

Obviously, Lemma 5.5 shows that in the case when the Riemann Hypothesis holds and there are multiple non-trivial zeros, Theorem 1.4 is true even in a much stronger form.

5.2. The case of simple zeros under the Riemann Hypothesis. Assuming the Riemann Hypothesis and the simplicity of the non-trivial zeros we write

$$
G(z)=\sum_{\gamma>0} \frac{\zeta^{2}(\rho / 2)}{\rho \zeta^{\prime}(\rho)} e^{i \gamma z / 2}
$$

where $\Im(z)>0$. Assuming $(\mathrm{H})$ the series converges absolutely and uniformly on every compact subset of the upper half-plane and hence $G(z)$ is holomorphic for $\Im(z)>0$. The finer analytic properties of $G(z)$ are described in 21. In particular it easily follows from Theorems 3 and 4 of [21] that for positive real $x$ the limit $\lim _{y \rightarrow 0^{+}} \Re G(x+i y)$ exists and equals

$$
\frac{1}{2} e^{-x / 4} E_{2}\left(e^{x}\right)+O\left(\exp \left(-\frac{1}{4} x+O\left(\frac{x}{\log x}\right)\right)\right) .
$$

Moreover, by Lemma 4.1 in [11] for $z=-\log (4 m)+\delta e^{i \theta}, 0<\theta<\pi, 0<\delta<1$, $m \in \mathbb{N}$, we have

where

$$
G(z)=-\frac{a(m)}{2^{3 / 2} \pi m^{3 / 4}} \frac{1}{\delta^{1 / 2}} e^{-i \theta / 2}+O_{m}(1)
$$

$$
a(m)=\sum_{l^{2} \mid m} l \mu(l) d\left(m / l^{2}\right),
$$

$\mu$ and $d$ being the usual Möbius and divisor functions. In particular we see that $G$ belongs to the class $\mathfrak{A}$ with parameters $B=B^{*}+1$, where $B^{*}$ is as in (1.6), $L_{0}=0, x_{1}=-6 \log 2, x_{2}=-2 \log 2,0<\theta_{1}^{(j)}<\theta_{2}^{(j)}<\pi(j=1,2)$ arbitrary fixed, and $\phi(u)=u^{-1 / 2}$.

An application of Theorem 2.1 ends the proof of Theorem 1.4.

\section{REFERENCES}

1. R. C. Baker, The square-free divisor problem, Quart. J. Math. (Oxford), (2) 45 (1994), 269277. MR1295577 (95h:11098)

2. R. C. Baker, The square-free divisor problem II, Quart. J. Math. (Oxford), (2) 47 (1996), 133-146. MR.1397933 (97f:11080)

3. A.A. Gioia, A.M. Vaidya, The number of square-free divisors of an integer, Duke Math. J., 33 (1966), 797-799. MR0202678 (34:2538)

4. J. Kaczorowski, On sign-changes in the remainder-term of the prime-number formula, I, Acta Arith. 44 (1984), 365-377. MR.777013 (87c:11081a)

5. J. Kaczorowski, On sign-changes in the remainder-term of the prime-number formula, II, Acta Arith.45 (1985), 65-74. MR791085 (87c:11081b)

6. J. Kaczorowski, On sign-changes in the remainder-term of the prime-number formula, III, Acta Arith. 48 (1987), 347-371. MR927376 (89d:11074)

7. J. Kaczorowski, On sign-changes in the remainder-term of the prime-number formula, IV, Acta Arith. 50 (1988), 15-21. MR945273 (89k:11081)

8. J. Kaczorowski, J. Pintz, Oscillatory properties of arithmetical functions, I, Acta Math. Hungar. 48 (1-2)(1986), 173-185. MR858395 (88c:11053) 
9. J. Kaczorowski, The $k$-functions in multiplicative number theory, I, On complex explicit formulae, Acta Arith. 56 (1990), 195-211. MR1083000(91m:11068a)

10. J. Kaczorowski, The k-functions in multiplicative number theory, IV; On a method of A.E. Ingham, Acta Arith. 57 (1991), 231-244. MR1105608 (92f:11117b)

11. J. Kaczorowski, K. Wiertelak, $\Omega$-estimates for a class of arithmetic error terms, Math. Proc. Camb. Phil. Soc. 142(2007), 385-394. MR2329690(2008c:11126)

12. J.E. Littlewood, Sur la distribution des nombres premiers, C.R. Acad. Sci. Paris 158 (1914), $1869-1872$.

13. F. Mertens, Über einige asymptotische Gesetze der Zahlentheorie, J. reine angew. Math. $\mathbf{7 7}$ (1874), 289-338.

14. H. L. Montgomery, The zeta function and prime numbers, Proceedings of the Queen's Number Theory Conference, 1979 (Kingston, Ont., 1979), pp. 1-31, Queen's Papers in Pure and Appl. Math., 54, Queen's Univ., Kingston, Ont., 1980. MR634679 (82k:10047)

15. H.L. Montgomery, U.M.A. Vorhauer, Changes of sign of the error term in the prime number theorem, Functiones et Approximatio, 35 (2006), 235-247. MR 2271616 (2008c:11123)

16. W.G. Nowak, M. Schmeier, Conditional asymptotic formulae for a class of arithmetic functions, Proc. Amer. Math. Soc. 103 (1988), 713-717. MR947644 (89f:11130)

17. J. Pintz, Cramér vs. Cramér. On Cramér's probabilistic model for primes, Functiones et Approximatio, 37(2) (2007), 361-376. MR2363833

18. B. Saffari, Sur le nombre des diviseurs $r$-libres d'un entier, et sur les points à coordonnées entières dans certaines régions du plan, C.R. Acad. Sci. Paris Sér. A-B 266 (1968), A601A603. MR0230692 (37:6252)

19. D. Suryanarayana, V. Siva Rama Prasad, The number of $k$-free divisors of an integer, Acta Arith. 17 (1970/71), 345-354. MR0327697 (48:6039)

20. E. C. Titchmarsh, The theory of the Riemann zeta-function, Second edition, revised by D. R. Heath-Brown, Oxford, 1986. MR882550 (88c:11049)

21. K. Wiertelak, On some connections between zeta-zeros and square-free divisors of an integer, Functiones et Approximatio, 31 (2003), 133-145. MR2059542 (2005b:11136)

22. Wu, Jie, On the primitive circle problem, Monatsh. Math., 135 (2002), 69-81. MR.1894296 (2003a:11103)

Faculty of Mathematics and Computer Science, Adam Mickiewicz University, 61-614 Poznań, Poland.

E-mail address: kjerzy@amu.edu.pl

Faculty of Mathematics and Computer Science, Adam Mickiewicz University, 61-614 Poznań, Poland.

E-mail address: wiertela@amu.edu.pl 